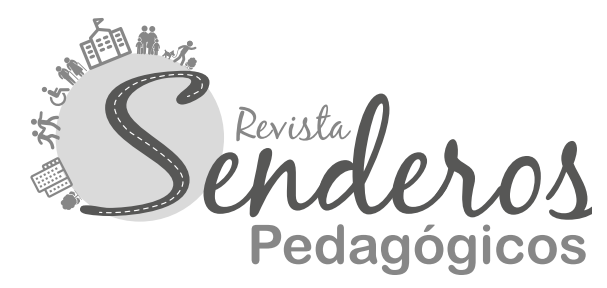

\title{
Escritura académica: errores frecuentes en artículos postulantes para la Revista Senderos Pedagógicos ${ }^{1}$
}

\author{
Academic writing: frequent errors in candidate articles for the \\ Senderos Pedagógicos Journal
}

\author{
Autora: \\ Astrid Efigenia Isaza Jaramillo \\ https://orcid.org/oooo-0003-1890-5468 \\ Recibido: $24 / 11 / 2020$ \\ Aprobado: 26/08/2021
}

1 Artículo de reflexión no derivado de investigación.

2 Licenciada en Educación Básica con Énfasis en Humanidades y Lengua Castellana, Tecnológico de Antioquia - Institución Universitaria. astriefi@gmail.com.

\section{Resumen:}

Este artículo de reflexión no derivado de investigación tiene como propósito dar a conocer los errores más frecuentes de redacción, coherencia, ortografía y uso incorrecto de citaciones que se evidenciaron en los textos postulados para la décimo primera edición de la Revista Senderos Pedagógicos. La reflexión surge a partir de la experiencia como Asistente editorial en la ejecución de la Práctica profesional II de la Licenciatura en Educación Básica con Énfasis en Humanidades y Lengua Castellana del Tecnológico de Antioquia - Institución Universitaria; práctica realizada durante el segundo semestre del año 2020.

Palabras clave: escritura académica, corrección de estilo, edición de textos.

\begin{abstract}
:
The purpose of this reflection article not derived from research is to make known the most frequent errors in writing, coherence, spelling and incorrect use of citations that were evidenced in the texts
\end{abstract}


postulated for the eleventh edition of the Senderos Pedagógicos Journal. The reflection arises from the experience as Editorial Assistant in the execution of Professional Practice II of the Bachelor's Degree in Basic Education with an Emphasis on Humanities and Spanish
Language of Tecnológico de Antioquia

- University Institution; practice carried out during the second semester of 2020 .

Keywords: academic writing, style correction, text editing.

\section{Introducción}

La Revista Senderos Pedagógicos (Resep) es un medio de divulgación y difusión nacional e internacional del conocimiento de las ciencias sociales, en el área de las ciencias de la educación, adscrita a la disciplina de educación general. La revista es liderada por el Grupo de Investigación Senderos de la Facultad de Educación y Ciencias Sociales del Tecnológico de Antioquia - Institución Universitaria. La periodicidad de publicación es anual. Es editada en versión impresa y digital, con acceso libre y gratuito para lectores y articulistas. El objetivo de Resep es contribuir a la construcción de nuevo conocimiento desde el área de la educación y servir de plataforma para la divulgación y visibilización de investigadores emergentes y consolidados, interesados en aportar a la comprensión y solución de problemas de las ciencias sociales desde el campo de la educación y la pedagogía (Revista Senderos Pedagógicos, 2020).

Desde su inicio en el 2010, esta publicación se ha caracterizado por dar a conocer investigaciones relacionadas con las infancias, las pluralidades y la gestión educativa. En esta medida, la revista se ha ido fortaleciendo con artículos resultado de investigaciones que enriquecen la discusión, tanto de investigadores, como de personas que se forman en temas afines a nivel de pregrado y posgrado.

A la fecha se han dado a conocer diez ediciones en versión impresa y digital, con acceso libre y gratuito para lectores y articulistas. La revista abre convocatorias permanentes, con un cronograma definido para cada número; es una publicación científica arbitrada con pretensiones de indexación, por lo cual privilegia las siguientes modalidades de artículos: investigación científica y tecnológica, reflexión y revisión; además, se consideran: artículo corto, reporte de caso, revisión de tema, carta del editor, reseñas, entrevistas, ponencias, artículo de reflexión no derivado de investigación y artículo de opinión (Revista Senderos Pedagógicos, 2020).

Desde esa perspectiva, esta reflexión surgió desde la observación de los errores más frecuentes de redacción, coherencia, ortografía y uso incorrecto de citaciones que presentaron los artículos postulados para la décimo primera edición de Resep, lo que implica tener en consideración una serie de lineamientos 
establecidos por la misma. En este sentido, este texto presenta de forma organizada, la importancia de publicar artículos científicos y la significación de la escritura académica.

\section{Metodología}

Durante la ejecución de la Práctica profesional II de la Licenciatura en Educación Básica con Énfasis en Humanidades y Lengua Castellana, se revisaron nueve (9) artículos postulados para la décimo primera edición de Resep. La metodología implementada en las observaciones fue a partir de un diseño de rastreo, en el que se determinó el cumplimiento de los requisitos para que el artículo continuara o no con el proceso de edición según las orientaciones del Editor y el Comité editorial ${ }^{3}$.

Para esta revisión se utilizó el formato de "Rejilla previa de evaluación previa” que determina un listado de requisitos para validar las postulaciones en la etapa de inicio; formato que establece:

- Información general: título del artículo, autor, tipo de artículo, fecha de recepción, fecha de revisión, pertinencia del artículo para la revista desde el criterio editorial.

- Cumplimiento de requisitos de formato: Word, tamaño de letra, pie de página, interlineado, espaciado, citas textuales y contextuales.

- Estructura: extensión del texto, extensión del título, resumen, palabras clave, traducción al idioma inglés tanto del resumen como de las palabras clave, escritura del artículo según la estructura solicitada por Resep.

- Referencias: cumplimiento de las referencias en concordancia al estilo de la American Psychological Association (APA), prueba de originalidad a partir de la verificación de autenticidad en el software Turnitin; firma del Editor y del Asistente editorial (Revista Senderos Pedagógicos, 2020).

De acuerdo al cumplimiento de cada uno de los requerimientos editoriales, el Editor y el Comité editorial determinan la pertinencia de la propuesta para que continúe con las próximas valoraciones; de lo contrario, se devuelve el texto

3 El equipo editorial de la décimo primera edición es el siguiente: Comité científico: Olga Patricia Ramírez Otálvaro, María Gladys Romero Quiroga, Norelly Soto Builes, Claudia Vélez de la Calle, Martha Vergara Fregoso; Comité editorial: Tatiana Goldrine Godoy, Eliana Verónica Romo López, Libia Vélez Latorre, Beatriz Elena Zapata Ospina; Director: Jorge Iván Correa Alzate; Editora: Carolina Moreno Echeverry; Asistente editorial: Astrid Efigenia Isaza Jaramillo; Fotógrafo: Galo Ibarra Palacios. 
al autor señalando los motivos de rechazo. Posteriormente, el Editor envía el manuscrito a los pares evaluadores, dictamen en el que el árbitro seleccionado decide sobre la publicación del artículo que podrá ser: aceptar el artículo para publicar sin modificaciones, aceptar el artículo sin cambios sustantivos, aceptar el artículo con modificaciones mayores, no publicar el artículo (Revista Senderos Pedagógicos, 2020).

Para llevar a cabo el dictamen, el par evaluador tiene en cuenta las siguientes pautas definidas en la "Guía de evaluación - Artículo resultado de investigación":

- Información del par evaluador: nombre, documento de identificación, ciudad, país, teléfono de contacto, correo electrónico, título de pregrado y posgrado, filiación institucional, dirección de correspondencia, Currículum Vitae Latinoamericano y del Caribe (CvLAC).

- Aceptación del código de ética por parte del evaluador: revisión de trabajos que correspondan a su formación académica y áreas de especialidad; seguir el mecanismo de revisión de los manuscritos basado en el método de doble ciego internacional, garantizando los estándares de anonimato; respetar el sistema de revisión doble ciego; realizar una revisión cuidadosa, analítica, objetiva e imparcial del artículo, ajustándome al formato de valoración de Resep; respetar el criterio de confidencialidad del dictamen de la evaluación; no realizar ningún tipo de distribución ni difusión de los trabajos evaluados; no obtener beneficios personales o apropiarse del contenido de los artículos evaluados; emitir comentarios al Comité editorial en caso de considerar que existe algún conflicto de tipo ético o de respeto por la propiedad intelectual que entren en conflicto en el material recibido.

- Información del artículo: título del artículo, tipo de artículo (resultado de investigación, reflexión o revisión) fecha de recepción por el par evaluador, fecha de envío por el par evaluador al Comité editorial.

- Valoración del artículo: se consideran las siguientes preguntas orientadoras:

- ¿El título es conciso y da cuenta del contenido esencial del artículo?

- ¿El resumen del artículo comunica de manera clara el contenido del artículo, dando cuenta de cada uno de los apartes (planteamiento del problema, objetivo, metodología, resultados y discusión y conclusiones)?

- ¿La introducción plantea de forma coherente el contenido del artículo? 
- ¿El artículo cumple con el rigor metodológico tanto en su estructura como en la pertinencia del método utilizado?

- ¿Los resultados, discusiones y conclusiones son coherentes?

- ¿El trabajo es una contribución al campo específico que analiza? Valoración técnica y redacción: se tienen en cuenta los siguientes criterios:

- ¿Considera que el estilo de escritura cumple con los criterios de rigurosidad científica?

- ¿El lenguaje utilizado es claro, conciso y facilita la lectura?

- ¿La bibliografía utilizada es adecuada?

- Otras observaciones y comentarios para el autor: ¿considera que existe algún aspecto de tipo ético o de respeto por la propiedad intelectual que entren en conflicto en el artículo?

- Otras observaciones y comentarios para el autor: en este apartado, el par evaluador consigna las observaciones, sugerencias y modificaciones que debe tener en cuenta el autor para que la propuesta pueda ser publicada.

- Concepto final: en este campo, el par dictamina si se publica o no el artículo, o en su defecto establece las modificaciones a realizar para que este pueda darse a conocer (Revista Senderos Pedagógicos, 2020).

En caso de que el artículo sea aceptado, condicionado a la realización de modificaciones y ajustes, el Editor consulta al autor la voluntad de continuar con el proceso editorial. En caso de obtener una respuesta negativa por parte del autor, el artículo se entiende rechazado. De resultar positiva la respuesta del autor, se concede un nuevo término para realizar los ajustes y modificaciones, y devolver la nueva versión del escrito a el Editor de la revista. En caso de no estar de acuerdo con algunas de las recomendaciones puede argumentarlo por medio de una carta al Editor. Una vez revisadas las modificaciones se informa el resultado al autor. La decisión final de publicar o rechazar los trabajos depende directamente del Comité editorial (Revista Senderos Pedagógicos, 2020).

Según las nuevas versiones entregadas por los autores, se realizaron lecturas comparadas de los artículos que fueron revisadas por el par evaluador de acuerdo con los criterios de evaluación establecidos en la "Rejilla previa de evaluación previa” y la “Guía de evaluación - Artículo resultado de investigación”; además de los comentarios sobre la escritura del texto, tanto de forma como de contenido. En estas verificaciones, se realizó un contraste detallado y minucioso de acuerdo a las sugerencias establecidas por el par y las correcciones ejecutadas por el autor. En ese sentido, se procedió a indicar si en realidad se llevaron a cabo las recomendaciones o, por el contrario, el artículo no cumplía con lo establecido por la revista para ser publicado. 
Con base en lo anterior, una vez comprobado que en efecto el autor cumplió con las recomendaciones del par evaluador, se procedió a realizar los ajustes, y así el Editor y el Comité editorial se encargaban de tomar las decisiones finales de publicación.

Ahora bien, para la presentación de los aportes a esta reflexión se tuvieron en cuenta los errores más frecuentes de los artículos revisados en relación con la coherencia, cohesión y el uso adecuado de normas APA. Además, el contenido de esta reflexión está soportado en argumentos de autoridad de expertos en el tema, que contribuyen a mejorar la escritura académica para las personas interesadas en postular un artículo para la Resep.

\section{Importancia de publicar y escribir artículos académicos}

Uno de los propósitos de la investigación científica es publicar; por consiguiente, difundir artículos académicos e investigativos en revistas indexadas es de gran importancia para quienes profesan y se inician en la investigación. A esto se suma que uno de los objetivos de las labores científicas es promover el acceso a la investigación, posibilitando la circulación de conocimiento:

Los hombres y mujeres de ciencia cuando comienzan como estudiantes graduados, no son juzgados principalmente por su habilidad en los trabajos de laboratorio, ni por su conocimiento innato de temas científicos, ni, desde luego, por su ingenio o encanto personal; se los juzga y se les conoce por sus publicaciones (Day, 2005, p. 9).

Esto indica que, publicar textos académicos le proporciona al autor un reconocimiento en el mundo editorial y, por supuesto, en el campo universitario. Por lo tanto, publicar artículos establece resultados disciplinares favorables para la sociedad. Túñez et al. (2014), destaca “[...] el incentivo al trabajo conjunto entre autores y revistas para alcanzar la visibilidad de los artículos en distintos medios de divulgación" (p. 6). En este sentido, algunas de las causas más reiteradas para difundir las investigaciones son:

- Estimulaciones desde la comunidad académica.

- Proyección de metas investigativas a nivel profesional.

- Desde una postura institucional, la elaboración de artículos ayuda a la obtención de registros calificados y acreditaciones de calidad. 
- De acuerdo a la cantidad y calidad de artículos publicados en revistas indexadas, favorece al autor en cuanto a la asignación de becas y recursos para próximas investigaciones.

- Las investigaciones entran a formar parte de comunidades científicas.

- Desde el campo laboral, se generan más oportunidades desde el medio editorial.

Dicho lo anterior, es necesario tener en cuenta que para llevar a cabo un proceso de publicación es muy importante "dar a conocer lo que se ha descubierto o comprendido (previa evaluación), con el fin de propiciar el diálogo entre el conocimiento y la sociedad" (Sánchez, 2011, p. 13). Situación que demanda un uso adecuado de la escritura, puesto que una de las características propias de la redacción es la claridad que se le proyecta al lector en lo que se quiere comunicar.

Según Day (2005), “[...] un artículo científico publicado resulta inútil si no es recibido y entendido por el público al que se destina” (p. 17). De ahí radica la importancia de escribir adecuadamente un texto académico e investigativo, puesto que se le está entregando a la comunidad científica y académica el resultado coherente de una investigación que puede ser referente de estudio para próximos investigadores; lo que significa que, si no hay un lenguaje sencillo, el lector no logrará conectarse con lo que se quiso establecer en el texto:

Sencillamente, la ciencia es demasiado importante para ser
comunicada de cualquier otra forma que no sea con palabras de
significado indudable. Ese significado indudable y claro debe serlo
no solo para colegas del autor, sino también para los estudiantes que
acaban de iniciar su carrera, para los científicos de otras disciplinas y,
especialmente, para los lectores cuya lengua nativa no es la misma del
autor (p. 18).

Si bien, el resultado final de una investigación equivale a la publicación, no se debe dejar a un lado todo lo que se requiere para publicar un artículo. Un autor puede tardar meses e incluso años en la recolección de datos; este arduo trabajo puede correr el riesgo de no lograr el objetivo de comunicación si el productor no es claro y específico con el lenguaje que desea transmitir. Parafraseando a Day (2005), en la redacción científica el lenguaje debe ser lo más claro y sencillo posible, así se difunde el sentido con el menor número posible de palabras. El estilo debe ser el más apropiado para cumplir esa labor; de esa manera, se logra establecer un vínculo comunicativo con el receptor. 


\section{Errores frecuentes en artículos postulantes}

Durante la ejecución de la práctica se revisaron nueve (9) propuestas, desde la modalidad de artículos resultados de investigación, de reflexión y reporte de caso. Es muy usual que en el proceso de escritura intervengan dificultades lingüísticas, lo que ocasiona que el nivel intencional de comunicación no sea el esperado; como consecuencia de ello, se devuelve el artículo por parte del Editor y el Comité editorial de la revista. En palabras de Day (2005), “[...] si no se tiene un propósito claro, puede ocurrir que se escriba en seis direcciones distintas al mismo tiempo" (p. 32). En efecto, algunos de los errores encontrados en la redacción, coherencia, ortografía y el uso incorrecto en la citación, contribuyen que desde una primera lectura se generen confusiones que, sin lugar a dudas acarrea una devolución al autor por causa de no tener claridad en el propósito o en la intencionalidad del texto.

La corrección de estilo juega un papel muy importante dentro del proceso editorial, puesto que garantiza la calidad del contenido que se va a publicar. Este ejercicio de revisión es llevado a cabo por un corrector profesional, quien determina desde una lectura detallada imprecisiones de vocabulario y evita algunas muletillas en la expresión del escritor. Parafraseando a la agencia literaria Letras Propias (s.f.), un texto bien terminado y liberado de errores evidentes habla mucho de quien lo escribe; no solo se evidencia el esfuerzo del autor, sino el respeto y el valor que el lector aprecia del mismo.

En consonancia, la corrección de estilo consta de dos vertientes. La primera tiene que ver con la estructura o forma (ortografía y sintaxis); y la segunda, desde el contenido o fondo (semántica, retórica y estilística). Dentro de las funciones realizadas como Asistente editorial de Resep, los errores observados en las propuestas investigativas están relacionados con la forma.

Zavala (2012) manifiesta que la expresión "corrección de estilo" no es exacta, pues el término ha sido más usado por la falta de hallar un modismo más adecuado, ya que la palabra estilo se refiere a algo más personal que nadie puede corregir. Más allá de este planteamiento, el corrector de estilo se encarga de perfeccionar escritos originales para que estos puedan publicarse. Llegados a este punto, la función de un corrector de estilo se encuentra relacionada con la inspección ortográfica desde el uso correcto de los signos de puntuación, coherencia, cohesión y la adecuada escritura de la lengua.

De acuerdo a lo anterior fue muy usual encontrar errores ortográficos en la revisión de los artículos de la décimo primera edición, entre los más frecuentes están: uso incorrecto de la coma, generalmente separando el sujeto del verbo. Según la Real Academia Española de la Lengua (RAE) (s. f.): "Es incorrecto escribir coma entre el sujeto y el verbo de una oración, incluso cuando el sujeto 
está compuesto de varios elementos separados por comas". Si bien, el uso de la coma también tiene que ver con el gusto o la intención comunicativa de la persona que escribe, hay algunas excepciones que obligan su ubicación para la comprensión del lector y, de esa manera, evitar confusiones en el mensaje que se quiere transmitir.

A esto se suma la coherencia, cohesión, repetición de los mismos conectores textuales (así mismo, en ese sentido, por lo cual, por las cuales, en el cual, sin embargo), y la falta de uso de sinónimos en palabras como: religioso, aprendizaje, etapa, comportamiento; siendo estas algunas de las fallas más observadas en la revisión de los textos presentados a la revista. Si un texto no es coherente, significa que no hay claridad para el lector; para ello, se hace necesario establecer un buen uso de sinónimos y conectores que ayuden a enriquecer la escritura desde la estructura propia de los párrafos, como del contenido en general. Lara (2011) acuña que “[...] la disciplina que enseña a ordenar en forma lógica estas oraciones es la gramática. Así mismo, existe otra rama que ayuda a comunicar mejor las cosas: la ortografía. Gracias a ella, se puede utilizar bien el lenguaje" (p. 132). El correcto uso sintáctico, morfológico y semántico en la construcción de un texto permite que el lector pueda hallar el significado global de este.

En términos de estructura, algunos de los artículos de la décimo primera edición presentaron anomalías con la escritura del resumen, metodología, conclusiones, citación, referencias y bibliografía. En tanto que era muy frecuente encontrar incoherencias en el contenido de los textos. A continuación, se dará a conocer en detalle cada una de las inconsistencias mencionadas anteriormente.

\section{Resumen}

En relación al resumen, si bien, en este se debe dar a conocer de una manera breve el contenido de la investigación y hacia dónde se quiere llegar con la misma, fue muy usual encontrar resúmenes que no eran coherentes con el contenido. Por su parte, Sánchez (2011) manifiesta lo siguiente:

Un resumen bien elaborado es el párrafo más importante del artículo, y la primera información que evaluarán los editores y los árbitros. Permite que los lectores reconozcan con rapidez el contenido. El resumen, es una presentación abreviada y precisa del contenido de un documento, en el cual se debe evitar incluir interpretación o crítica (p. 154). 
Es por esto que, al momento de diseñar el resumen, es muy importante seleccionar la información más relevante y omitir aquella de poca trascendencia en el texto. Parafraseando a Day (2005), el resumen debe dar claridad de los objetivos, nombrar los métodos empleados, mostrar los resultados y por supuesto enunciar las conclusiones más importantes.

\section{Metodología}

En cuanto a la metodología, se encontraron algunas inconsistencias relacionadas con el lugar, tiempo, la manera en cómo se llevó a cabo la investigación y los materiales utilizados. Este apartado es muy importante en el contenido del artículo debido a que describe detalladamente cómo se realizó el estudio de la investigación. Es decir, características de los participantes que constituyen la muestra, especificar el contexto y el medio en el que se lleva a cabo la investigación, los métodos de recolección de datos (entrevistas, test, talleres grupales, etcétera) y las técnicas de análisis empleadas. "El objetivo de la metodología es brindar la información necesaria para que el lector juzgue la validez y confiabilidad de los resultados" (Centro de Escritura Javeriano, 2020).

\section{Conclusiones}

Desde las conclusiones se interpretó que en algunos casos no tenían concordancia con los objetivos establecidos; además, fue muy frecuente encontrar citas en el desarrollo de las mismas. Las citas son fundamentales, puesto que le aportan solidez al contenido del trabajo; sin embargo, se recomienda no utilizarlas en las conclusiones, debido a que, en este momento del texto, el autor presenta una perspectiva crítica de la investigación; es decir, si se cumplieron o no los objetivos, comparaciones con los resultados de otros trabajos, además de recomendaciones para nuevas investigaciones.

Las conclusiones deben ser breves y deben precisar las aportaciones al conocimiento apoyadas en los resultados del propio trabajo, no de investigaciones ajenas. Además, no se basan en apreciaciones subjetivas, no se enumeran y no llevan abreviaturas, sino términos completos. Es necesario comprender que debe haber coherencia entre estas y la información descrita en el resumen. 


\section{Citas}

Al mismo tiempo, en todas las propuestas se hallaron errores en el momento de citar a un autor o autores, entre los más repetitivos fueron:

- Tipo y tamaño de letra en citas de más de 40 palabras.

- Falta de paginación en citas cortas textuales.

- Confusión con el parafraseo y cita textual.

- Ausencia del año.

- Citas no coherentes con el contenido del trabajo.

El Centro de Escritura Javeriano (2020) afirma que “[...] las citas corresponden a las ideas que se retoman de otras fuentes de información de un texto, con una correcta atribución de los créditos de autoría” (p. 12). El estilo establecido por APA determina que en el momento de citar se debe considerar el tipo de cita que se quiere resaltar; es decir, cita textual (corta, larga), en la que se plasma la información tal cual como la designan los autores, y cita parafraseada, tipo de mención que explica lo que quiere decir el autor con palabras propias sin perder la esencia original.

\section{Referencias y bibliografías}

En relación a las referencias y bibliografías, generalmente las postulaciones no cumplen con la estructura solicitada por las normas APA. De acuerdo con el Centro de Escritura Javeriano (2020):

Las referencias son un listado con la información completa de las fuentes citadas en el texto. Son necesarias para la atribución correcta de los créditos de autoría y la localización y confirmación de la información en el caso de que un lector quiera acudir a las fuentes que sustentaron un trabajo (p. 22).

Esto quiere decir que todas las citas utilizadas en el texto deben estar referenciadas y todas las fuentes referenciadas deben ser nombradas en el mismo. También es muy importante tener en cuenta que este listado debe ubicarse en una hoja aparte una vez finalizado el escrito; además, el título debe ir centrado y en negrita, los autores se ubican en orden alfabético, se utiliza sangría francesa de 1,27 cm en la segunda línea y un interlineado de 2,o. 
Entre los errores más apreciados en los textos se encuentran:

- Tamaño de letra.

- Título sin cursiva.

- Ausencia de la sangría francesa en la segunda línea.

- Ausencia del espaciado doble.

- Citas no referenciadas.

Tener en cuenta este tipo de fallas sintácticas y gramaticales a la hora de enfrentarse con la escritura académica favorecerá en gran medida la publicación de un artículo; además, los niveles lingüísticos alcanzados permitirán un reconocimiento de indexación en la editorial postulante.

\section{Conclusiones y recomendaciones}

Escribir no es una tarea fácil y más aún cuando se requiere de la comprensión del lector para lo que se quiere comunicar. La escritura académica ha permitido que muchas personas den a conocer sus investigaciones y que, a partir de estas, desde la academia se fortalezcan los procesos de aprendizajes y de escritura para aquellos que quieren incursionar en ese medio. Para nadie es un secreto que llegar a ser reconocido desde el ámbito editorial es un trabajo arduo y de dedicación; no solo desde lo actitudinal, sino desde las habilidades lingüísticas que le facilitan el camino al interesado en publicar, puesto que para ello es necesario pulirse en este campo para lograr las metas propuestas.

Para publicar un artículo en una revista se requiere de un proceso detallado de verificación, tanto de forma como de contenido, de ahí la importancia de tener en cuenta los parámetros establecidos por la revista para evitar contratiempos y devoluciones en la etapa inicial del proceso. Desde esa perspectiva y de acuerdo a los errores más frecuentes hallados en las revisiones de los artículos con intereses de publicación, se sugiere que una vez se inicie con la escritura de este tipo de textos, el autor debe tener a la mano una lista de conectores lógicos que le permitan unir las ideas de una manera clara y coherente; así, el texto empieza a tener un estilo adecuado y un lenguaje atractivo para futuros lectores. A esto se suma tener como referentes diccionarios de la RAE, tales como: americanismos, palabras comunes de la lengua castellana, panhispánico de dudas, entre otros, que le ayudarán a mejorar la redacción y cohesión en el escrito.

Tener en cuenta este tipo de estrategias facilitarán el proceso de edición; así el interesado también se evitará devoluciones y ahorrará tiempo en modificaciones que posiblemente pueden ser un detonante para desistir de la 
publicación. Si bien no es un camino fácil de recorrer, publicar trae consigo una serie de reconocimientos tanto del mundo editorial, como del académico.

En definitiva, si dentro de las aspiraciones académicas se encuentran publicar un artículo, no hay que olvidar lo dicho en este artículo de reflexión no derivado de investigación. Seguramente muchas personas consideran que publicar es solo para los expertos; sin embargo, es importante tener en cuenta que este camino puede ser transitado por todos aquellos que deseen iniciarse en el camino de la investigación; la diferencia está en que se requiere disciplina, compromiso, práctica y fortalecer permanente las habilidades lingüísticas.

\section{Referencias}

American Psychological Association (APA). https://www.apa.org/

Centro de Escritura Javeriano. (2020). Normas APA, séptima edición. Pontificia Universidad Javeriana, seccional Cali. https://www2.javerianacali.edu.co/centroescritura/recursos/manual-de-normas-apa-septima-edicion\#gsc.tab=0\%C2\%Ao.

Day, R. (2005). Cómo escribir y publicar trabajos científicos. Organización Panamericana de la Salud. http://acreditacion.unillanos.edu.co/CapDocentes/contenidos/ actualizacion_sep_2013/capacitacion_docente/produccion_textual/trabajos_ cientificos.pdf.

Lara, M. (2011). Fundamentos de investigación un enfoque por competencias. Alfaomega.

Letras propias. (s.f.). Corrección de textos. http://letraspropias.com/correccionortotipografica-y-de-estilo/.

Real Academia Española. (s. f.). Diccionario panhispánico de dudas. https://www.rae.es/dpd/ respecto.

Revista Senderos Pedagógicos. (2020). Orientaciones para los autores. Revista Senderos Pedagógicos / ISSN: 2145-8243 / E-ISSN: 2590-8456. https://ojs.tdea.edu.co/index. php/senderos/about/submissions.

Sánchez, A. (2011). Manual de redacción académica e investigativa: cómo escribir, evaluar y publicar artículos. Católica del Norte Fundación Universitaria.

Túñez, M., Valerezo, K., Marín, I. (2014). Impacto de la investigación y de los investigadores en comunicación en Latinoamérica: el índice h de las revistas científicas. Palabra Clave. Universidad de la Sabana. https://minciencias.gov.co/sites/default/files/upload/ noticias/120816-vfpolitica_publindex_2.o_og_ao_miv.pdf.

Zavala, R. (2012). El libro y sus orillas: tipografía, originales, redacción, corrección de estilo y de pruebas. Fondo de Cultura Económica. 


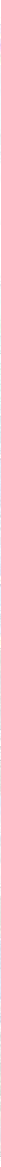

